\title{
A NOTE ON HADAMARD TYPE INTEGRAL INEQUALITIES INVOLVING SEVERAL LOG-CONVEX FUNCTIONS
}

\author{
B. G. PACHPATTE
}

\begin{abstract}
In this note, two new inegral inequalities of Hadamard type involving several differentiable log-convex functions are given. Two refinements of Hadamard's integral inequality for log-convex functions recently established by Dragomir are shown to be recaptured as special instances.
\end{abstract}

\section{Introduction.}

The following inequality is well known in the literature as Hadamard's inequality (see $[5, \mathrm{p} .137])$ :

$$
f\left(\frac{a+b}{2}\right) \leq \frac{1}{b-a} \int_{a}^{b} f(x) d x \leq \frac{f(a)+f(b)}{2},
$$

where $f: I \rightarrow R$ is a convex function on the interval $I$ of real numbers and $a, b \in I$ with $a<b$. In [1] Dragomir and Mond have proved that the following inequalities of Hadamard type hold:

$$
\begin{aligned}
f\left(\frac{a+b}{2}\right) & \leq \exp \left(\frac{1}{b-a} \int_{a}^{b} \ln [f(x)] d x\right) \leq \frac{1}{b-a} \int_{a}^{b} G(f(x), f(a+b-x)) d x \\
& \leq \frac{1}{b-a} \int_{a}^{b} f(x) d x \leq L(f(a), f(b)) \leq \frac{f(a)+f(b)}{2}
\end{aligned}
$$

where $f: I \rightarrow(0, \infty)$ is a log-convex function, $G(p, q):=\sqrt{p q}$ is the Geometric mean and $L(p, q):=\frac{p-q}{\ln p-\ln q}(p \neq q)$ is the Logarithmic mean of the positive real numbers $p$, $q$ (for $p=q$, we put $L(p, p)=p$ ). Recently in [3] Dragomir has established the following interesting refinements of Hadamard's inequalities for log-convex functions.

Let $f: I \rightarrow(0, \infty)$ be a differentiable log-convex function on the interval of real numbers $\stackrel{0}{I}$ (the interior of $I$ ) and $a, b \in \stackrel{0}{I}$ with $a<b$. Then the following inequalities

Received and revised August 26, 2003.

2000 Mathematics Subject Classification. Primary 26D15, Secondery 26D99.

Key words and phrases. Hadamard type, integral inequalities, log-convex functions, Geometric mean, Logarithmic mean, Jensen's integral inequality. 
hold:

$$
\begin{aligned}
\frac{\frac{1}{b-a} \int_{a}^{b} f(x) d x}{f\left(\frac{a+b}{2}\right)} & \geq L\left(\exp \left[\frac{f^{\prime}\left(\frac{a+b}{2}\right)}{f\left(\frac{a+b}{2}\right)}\left(\frac{b-a}{2}\right)\right], \exp \left[-\frac{f^{\prime}\left(\frac{a+b}{2}\right)}{f\left(\frac{a+b}{2}\right)}\left(\frac{b-a}{2}\right)\right]\right) \\
& \geq 1
\end{aligned}
$$

and

$$
\begin{aligned}
\frac{\frac{f(a)+f(b)}{2}}{\frac{1}{b-a} \int_{a}^{b} f(x) d x} & \geq 1+\log \left[\frac{\int_{a}^{b} f(x) d x}{\int_{a}^{b} f(x) \exp \left[\frac{f^{\prime}(x)}{f(x)}\left(\frac{a+b}{2}-x\right)\right] d x}\right] \\
& \geq 1+\log \left[\frac{\frac{1}{b-a} \int_{a}^{b} f(x) d x}{f\left(\frac{a+b}{2}\right)}\right] \geq 1 .
\end{aligned}
$$

For some recent results related to the Hadamard's inequality, see the books $[2,4,5]$ where further references are given. The main purpose of this note is to establish the general versions of the inequalities (1.3) and (1.4) involving several differentiable logconvex functions. The method employed in our analysis is based on the basic properties of logarithms and the application of the well known Jensen's integral inequality.

\section{Main Results}

we start with the following theorem.

Theorem 1. Let $f_{i}: I \rightarrow(0, \infty)(i=1, \ldots, n)$ be differentiable log-convex functions on the interval of real numbers $\stackrel{0}{I}$ (the interior of $I$ ) and $a, b \in \stackrel{0}{I}$ with $a<b$. Then the following inequalities hold:

$$
\begin{aligned}
\frac{\frac{1}{b-a} \int_{a}^{b} \prod_{i=1}^{n} f_{i}(x) d x}{\prod_{i=1}^{n} f_{i}\left(\frac{a+b}{2}\right)} \geq & L\left(\exp \left[\sum_{i=1}^{n} \frac{f_{i}^{\prime}\left(\frac{a+b}{2}\right)}{f_{i}\left(\frac{a+b}{2}\right)}\left(\frac{b-a}{2}\right)\right],\right. \\
& \left.\exp \left[-\sum_{i=1}^{n} \frac{f_{i}^{\prime}\left(\frac{a+b}{2}\right)}{f_{i}\left(\frac{a+b}{2}\right)}\left(\frac{b-a}{2}\right)\right]\right) \geq 1 .
\end{aligned}
$$

Proof. Since $f_{i}(i=1, \ldots, n)$ are differentiable and log-convex on $\stackrel{0}{I}$, we have that i.e.,

$$
\log f_{i}(x)-\log f_{i}(y) \geq \frac{d}{d y}\left(\log f_{i}(y)\right)(x-y),
$$




$$
\log f_{i}(x)-\log f_{i}(y) \geq \frac{f_{i}^{\prime}(y)}{f_{i}(y)}(x-y),
$$

for all $x, y \in \stackrel{0}{I}$. Writing $(2.2)$ for $i=1, \ldots, n$, adding the resulting inequalities and using the properties of log it is easy to observe that

$$
\log \left[\frac{\prod_{i=1}^{n} f_{i}(x)}{\prod_{i=1}^{n} f_{i}(y)}\right] \geq \sum_{i=1}^{n} \frac{f_{i}^{\prime}(y)}{f_{i}(y)}(x-y)
$$

for all $x, y \in \stackrel{0}{I}$. From (2.3) we have

$$
\prod_{i=1}^{n} f_{i}(x) \geq\left(\prod_{i=1}^{n} f_{i}(y)\right) \exp \left[\sum_{i=1}^{n} \frac{f_{i}^{\prime}(y)}{f_{i}(y)}(x-y)\right],
$$

for $x, y \in \stackrel{0}{I}$. By taking $y=\frac{a+b}{2}$ in $(2.4)$ we get

$$
\frac{\prod_{i=1}^{n} f_{i}(x)}{\prod_{i=1}^{n} f_{i}\left(\frac{a+b}{2}\right)} \geq \exp \left[\sum_{i=1}^{n} \frac{f_{i}^{\prime}\left(\frac{a+b}{2}\right)}{f_{i}\left(\frac{a+b}{2}\right)}\left(x-\frac{a+b}{2}\right)\right], \quad x \in[a, b] .
$$

Integrating (2.5) over $x$ on $[a, b]$ and using Jensen's integral inequality for exp (.) functions, we have

$$
\begin{aligned}
\frac{\frac{1}{b-a} \int_{a}^{b} \prod_{i=1}^{n} f_{i}(x) d x}{\prod_{i=1}^{n} f_{i}\left(\frac{a+b}{2}\right)} & \geq \frac{1}{b-a} \int_{a}^{b} \exp \left[\sum_{i=1}^{n} \frac{f_{i}^{\prime}\left(\frac{a+b}{2}\right)}{f_{i}\left(\frac{a+b}{2}\right)}\left(x-\frac{a+b}{2}\right)\right] d x \\
& \geq \exp \left[\frac{1}{b-a} \int_{a}^{b} \sum_{i=1}^{n} \frac{f_{i}^{\prime}\left(\frac{a+b}{2}\right)}{f_{i}\left(\frac{a+b}{2}\right)}\left(x-\frac{a+b}{2}\right) d x\right]=1 .
\end{aligned}
$$

Now by evaluating the middle integral in (2.6) as in [3, p.529] with suitable modifications, we get the required inequality in (2.1). The proof is complete.

The following inequality also holds.

Theorem 2. Let $f_{i}$ be as in Theorem 1. Then the following inequalities hold:

$$
\frac{\int_{a}^{b}\left(\prod_{i=1}^{n} f_{i}(y)\right)\left[\sum_{i=1}^{n} \frac{f_{i}^{\prime}(y)}{f_{i}(y)}\left(\frac{a+b}{2}-y\right)\right] d y}{\int_{a}^{b}\left(\prod_{i=1}^{n} f_{i}(y)\right) d y}
$$




$$
\begin{aligned}
& \leq \log \left[\frac{\int_{a}^{b}\left(\prod_{i=1}^{n} f_{i}(y)\right) \exp \left[\left[\sum_{i=1}^{n} \frac{f_{i}^{\prime}(y)}{f_{i}(y)}\left(\frac{a+b}{2}-y\right)\right] d y\right]}{\int_{a}^{b}\left(\prod_{i=1}^{n} f_{i}(y)\right) d y}\right] \\
& \leq\left[\frac{\left(\prod_{i=1}^{n} f_{i}\left(\frac{a+b}{2}\right)\right)}{\frac{1}{b-a} \int_{a}^{b}\left(\prod_{i=1}^{n} f_{i}(y)\right) d y}\right] .
\end{aligned}
$$

Proof. by taking $x=\frac{a+b}{2}$ in the inequality (2.4) we have

$$
\prod_{i=1}^{n} f_{i}\left(\frac{a+b}{2}\right) \geq\left(\prod_{i=1}^{n} f_{i}(y)\right) \exp \left[\sum_{i=1}^{n} \frac{f_{i}^{\prime}(y)}{f_{i}(y)}\left(\frac{a+b}{2}-y\right)\right]
$$

for all $y \in[a, b]$. Integrating (2.8) over $y$ and using Jensen's integral inequality for $\exp ($. functions, we have

$$
\begin{aligned}
& (b-a)\left(\prod_{i=1}^{n} f_{i}\left(\frac{a+b}{2}\right)\right) \\
\geq & \int_{a}^{b}\left(\prod_{i=1}^{n} f_{i}(y)\right) \exp \left[\sum_{i=1}^{n} \frac{f_{i}^{\prime}(y)}{f_{i}(y)}\left(\frac{a+b}{2}-y\right)\right] d y \\
\geq & \int_{a}^{b}\left(\prod_{i=1}^{n} f_{i}(y)\right) d y \exp \left[\frac{\left.\int_{a}^{b}\left(\prod_{i=1}^{n} f_{i}(y)\right)\left[\sum_{i=1}^{n} \frac{f_{i}^{\prime}(y)}{f_{i}(y)}\left(\frac{a+b}{2}-y\right)\right] d y\right]}{\int_{a}^{b}\left(\prod_{i=1}^{n} f_{i}(y)\right) d y}\right] .
\end{aligned}
$$

From (2.9) we have

$$
\begin{aligned}
& \exp \left[\frac{\int_{a}^{b}\left(\prod_{i=1}^{n} f_{i}(y)\right)\left[\sum_{i=1}^{n} \frac{f_{i}^{\prime}(y)}{f_{i}(y)}\left(\frac{a+b}{2}-y\right)\right] d y}{\int_{a}^{b}\left(\prod_{i=1}^{n} f_{i}(y)\right) d y}\right] \\
& \leq \frac{\int_{a}^{b}\left(\prod_{i=1}^{n} f_{i}(y)\right) \exp \left[\sum_{i=1}^{n} \frac{f_{i}^{\prime}(y)}{f_{i}(y)}\left(\frac{a+b}{2}-y\right)\right] d y}{\int_{a}^{b}\left(\prod_{i=1}^{n} f_{i}(y)\right) d y}
\end{aligned}
$$




$$
\leq \frac{(b-a)\left(\prod_{i=1}^{n} f_{i}\left(\frac{a+b}{2}\right)\right)}{\int_{a}^{b}\left(\prod_{i=1}^{n} f_{i}(y)\right) d y}
$$

which is equivalent to

$$
\begin{aligned}
& \quad \frac{\int_{a}^{b}\left(\prod_{i=1}^{n} f_{i}(y)\right)\left[\sum_{i=1}^{n} \frac{f_{i}^{\prime}(y)}{f_{i}(y)}\left(\frac{a+b}{2}-y\right)\right] d y}{\int_{a}^{b}\left(\prod_{i=1}^{n} f_{i}(y)\right) d y} \\
& \leq \log \left[\frac{\left.\int_{a}^{b}\left(\prod_{i=1}^{n} f_{i}(y)\right) \exp \left[\sum_{i=1}^{n} \frac{f_{i}^{\prime}(y)}{f_{i}(y)}\left(\frac{a+b}{2}-y\right)\right] d y\right]}{\int_{a}^{b}\left(\prod_{i=1}^{n} f_{i}(y)\right) d y}\right] \\
& \leq \log \left[\frac{\left(\prod_{i=1}^{n} f_{i}\left(\frac{a+b}{2}\right)\right)}{\frac{1}{b-a} \int_{a}^{b}\left(\prod_{i=1}^{n} f_{i}(y)\right) d y}\right] .
\end{aligned}
$$

This is the desired inequality in (2.7) and the proof is complete.

It is interesting to note that by taking $i=1$ and $f_{1}=f$ in Theorem 1 we get (1.3). Further, by taking $i=1$ and $f_{1}=f$ in Theorem 2 and making the elementary calculations as in $[3$, p.530] we recapture the inequality (1.4).

\section{References}

[1] S. S. Dragomir and B. Mond, Integral inequalities of Hadamard type for Log-convex functions, Demonstratio Mathematica 31(1998), 354-364.

[2] S. S. Dragomir and C. E. M. Pearce, Selected Topics on Hermite-Hadamard Inequalities and Applications, RGMIA Monographs, Victoria University, 2000.

[3] S. S. Dragomir, Refinments of the Hermile-Hadamard integral inequality for Log-convex functions, RGMIA Research Report Collection 3(2000), 527-533.

[4] D. S. Mitrinovic, J. E. Pecaric and A. M. Fink, Classical and New Inequalities in Analysis, Kluwer Academic Publishers, Dordrecht 1993.

[5] J. E. Pecaric, F. Proschan and Y. L. Tong, Convex Functions, Partial Orderings and Statistical Applications, Academic Press, New York, 1991.

57, Shri Niketen Coloney, Near Abhinay Talkies, Aurangabad 431001 (Maharashtra), India.

E-mail: bgpachpatte@hotmail.com 